\title{
Talks
}

\section{Young scientist forum}

\section{S01-01}

\section{Electron-beam micropatterning of titanium surfaces to stimulate human mesenchymal stromal cells}

\author{
*S. Janßen ${ }^{1}$, S. Gach ${ }^{2}$, S. Neuss-Stein ${ }^{3,4}$, S. Rütten ${ }^{3}$, S. Kant ${ }^{5}$, I. Lauria ${ }^{1}$, S. Olschok ${ }^{2}$, U. Reisgen ${ }^{2}$, H. Fischer ${ }^{1}$ \\ ${ }^{I}$ RWTH Aachen University Hospital, Dental Materials and Biomaterials Research, Aachen, Germany \\ ${ }^{2}$ RWTH Aachen, Welding and Joining Institute, Aachen, Germany \\ ${ }^{3}$ RWTH Aachen University Hospital, Institute of Pathology, Aachen, Germany \\ ${ }^{4}$ Helmholtz Institute for Biomedical Engineering, Biointerface Group, Aachen, Germany \\ ${ }^{5}$ RWTH Aachen University Hospital, Molecular and Cellular Anatomy, Aachen, Germany
}

\begin{abstract}
A rapidly established and strong bonding of native bone to the surface of a hard tissue implant is required to guarantee its long-term success.
\end{abstract} One option to influence the body's reaction to a foreign material is by modifying its surface properties, e.g. its topography.

To improve osseointegration, we propose a novel approach to introduce microtopographical cues to well-established titanium alloy Ti6Al4V surfaces by electron-beam structuring. Using this technique, we generated micropatterns with discontinuous features of $20 \mu \mathrm{m}$ height and $30 \mu \mathrm{m}$ diameter at intervals of 80 to $240 \mu \mathrm{m}$, as has been verified by laser scanning microscopy, and modified the surface chemistry during the structuring process.

To assess the suitability of this method as structuring process for future implant surfaces, the micropattern's influence on cell responses was investigated in-vitro using human mesenchymal stromal cells (hMSC) under standard growth conditions and conditions promoting osteogenesis. Resazurin metabolization indicates an improved cell viability compared to the unstructured material. Antibodystainings against Vinculin and scanning electron microscopy revealed adhesion of hMSC to the micro-topographical features. The ability to influence the differentiation of hMSC has been ascertained by real-time PCR analysis of osteogenic markers, such as alkaline phosphatase and Runx 2 .

The proposed electron-beam method for micro-structuring of titanium surfaces holds great potential to improve the osseointegration of titaniumbased hard tissue implants. 


\title{
S01-02
}

\section{Three-dimensional and non-invasive monitoring of dental implants}

\author{
*A. Ingendoh-Tsakmakidis ${ }^{1}$, L. Nolte ${ }^{2}$, A. Winkel ${ }^{1}$, H. Meyer $^{2}$, A. Koroleva ${ }^{3}$, A. Shpichka ${ }^{4}$, T. Ripken ${ }^{2}$, A. Heisterkamp ${ }^{5}$, M. Stiesch ${ }^{1}$ \\ ${ }^{1}$ Hannover Medical School, Department of Prosthetic Dentistry and Biomedical Materials Science, Hannover, Germany \\ ${ }^{2}$ Laser Zentrum Hannover e.V., Industrial and Biomedical Optics Department, Hannover, Germany \\ ${ }^{3}$ Laser Zentrum Hannover e.V., Nanotechnology Department, Hannover, Germany \\ ${ }^{4}$ Sechenov First Moscow State Medical University, Moscow, Russian Federation \\ ${ }^{5}$ Leibniz Universitaet of Hannover, Institute of Quantum Optics, Hannover, Germany
}

In order to restore the function of missing teeth, the insertion of dental implants is widely applied (Belibasakis GN 2014). In some cases, a biofilm-induced peri-implant inflammation may occur (Dreyer H et al 2018). Thus, new strategies for antibacterial implant coatings, surface structures, and materials are developed (Winkel A et al 2015). Implant material testing has been performed using terminal experimental procedures with determined time points. In this case, any information of cell response between those time points is absent, thus, each time point requires one implant sample. Furthermore, the majority of those implant test systems are limited to a 2D analysis, whereas dental implants require a volumetric analysis due to their 3D geometry. We introduced a new method, in order to reduce the sample numbers and to enable a time resolved and 3D monitoring of cell reactions along the implant. We established a non-toxic LIVE/DEAD staining for live-cell imaging, which was needed to distinguish between live and dead cells. Moreover, a 3D peri-implant model including a titanium implant colonized by human gingival fibroblasts was assembled fulfilling all necessary requirements, for instance, model stability, cell biocompatibility, and optical clarity. In addition, we applied SLOT (scanning laser optical tomography) to monitor non-invasively the implant for a period of three days (Lorbeer RA et al 2011). The gradual appearance of dead cells from up to down along the implant was successfully observed after application of toxic chlorhexidine concentrations to the top of peri-implant model. The cell reaction could be monitored on the whole implant surface up to three days. To sum up, this novel 3D and non-invasive method will complement and expand possibilities of future implant testing. The opportunity provided by this method permits an in vitro and time resolved examination of whole dental implants prior in vivo investigation in animals or during clinical trials. 
S01-03

\title{
Investigations on the initiator-free photopolymerization of Poly $(N-$ isopropylacrylamide) (PNIPAAm) layers on polypropylene and their application for the cultivation of mesenchymal stem cells (MSCs)
}

\author{
*R. Duckstein ${ }^{1}$, A. Schulz ${ }^{2}$, K. Lachmann ${ }^{1}$, M. Jänsch ${ }^{1}$, J. C. Neubauer ${ }^{2}$, H. Zimmermann², M. Thomas ${ }^{1}$, C. P. Klages ${ }^{1,3}$ \\ ${ }^{I}$ Fraunhofer IST, Atmospheric Pressure Processes, Braunschweig, Germany \\ ${ }^{2}$ Fraunhofer IBMT, Medical Biotechnology, Sulzbach, Germany \\ ${ }^{3}$ TU BS, Institute for Surface Technology, Braunschweig, Germany
}

PNIPAAm is the best-known representative thermoresponsive polymer. Due to a sharp volume phase transition at a lower critical solution temperature (LCST) in the range of body temperature of such polymer layers on low-cost polyolefin surfaces reversible control of surface wettability and biocompatibility can be achieved. Here, polypropylene (PP) surfaces were equipped with very thin pNIPAAM layers with the aim of cultivating MSCs and then gently releasing them from the surface by slightly lowering the temperature below $32{ }^{\circ} \mathrm{C}$.

The coating process is usually carried out in solution using free-radical initiators. In contrast, this work has established a gas-phase process with a simple apparatus design that can be easily integrated into industrial processes. Furthermore, a radical initiator has been omitted, which ensures that there is no migration of toxic substances out of the layer. For this purpose, a continuous stream of NIPAAm vapor in a carrier gas was passed over the PP surface, irradiated permanently by UV light with a wavelength of $254 \mathrm{~nm}$. It was not necessary to pre-activate the PP surface to initiate the polymerization. This was not expected since acrylamides, when using low concentrations, are not believed to have self-initiating properties. For a better understanding of initiator-free photopolymerization of PNIPAAm, ATR-FTIR studies were performed in situ on PPcoated zinc selenide $(\mathrm{ZnSe})$ ATR crystals. Based on these investigations, the coating rate of PNIPAAm could be further optimized so that the cultivation of MSCs was demonstrated successfully on this surface. In addition, it could be shown that the adhered cells react by lowering the temperature from $37{ }^{\circ} \mathrm{C}$ to $22{ }^{\circ} \mathrm{C}$ with the reduction of their cell spreading area. Occasionally, cells also completely detached from the surface. In further experiments, the harvest of all cells is to be achieved. 


\title{
S01-04
}

\section{Low-biofouling coatings using photoreactive polymers}

\author{
*S. Braun ${ }^{1,2}$, C. Yoshikawa ${ }^{2,3}$ \\ ${ }^{I}$ RWTH Aachen University, Chemistry, Aachen, Germany \\ ${ }^{2}$ CSIRO, Clayton, Australia \\ ${ }^{3}$ NIMS, Tsukuba, Japan
}

Biomedical devices are often used in contact with tissues, blood and other body liquids. Biomolecules such as proteins, cells and bacteria would adhere immediately to the devices surface, causing biological responses, like inflammation, blood clotting, encapsulation, etc. In order to prevent such undesired biological responses, photoreactive polymer coatings have been developed in this work. Therefore, random copolymers of $N$-( 2 hydroxypropyl)acrylamide (HPA) and benzophenone acrylamide (BPA) have been synthesized by RAFT polymerization in varying compositions. Kinetic studies of the copolymerization were carried out using a Chemspeed SWING robotic synthetic platform. The copolymers containing 1, 5, and $10 \mathrm{~mol} \% \mathrm{BPA}$ were spin-coated or cast-coated on silicon wafers or tissue culture polystyrene dishes. The BPA in these copolymers is responsible for the photoinduced crosslinking which leads to covalent bonding to the substrates surface as well as in the polymer itself. UV irradiation time was optimized, with regards to the degree of crosslinking, by monitoring the BPA peak by UV/VIS-spectroscopy. The composition at the surface of the crosslinked coatings was characterized by X-ray photoelectron spectroscopy (XPS). Contact angle and ATR-IR measurements confirmed that the substrates were homogenously covered with the copolymers. The swollen degrees of the coatings in phosphate buffer saline were determined by ellipsometry. Young's moduli of the swollen coatings were evaluated by atomic force microscopy (AFM). Finally, we demonstrated that the stiffness of the coatings significantly affected cell attachment and the optimized coating prevented cell adhesions. 


\section{S01-05}

\section{Electrospinning of Conductive Gelatin-PANi for Cardiac Patch Applications}

*F. Ruther ${ }^{1}$, A. R. Boccaccini ${ }^{1}$, F. B. Engel ${ }^{2}$

${ }^{I}$ Friedrich-Alexander Universität Erlangen-Nürnberg, Institute of Biomaterials, Erlangen, Germany

${ }^{2}$ Friedrich-Alexander Universität Erlangen-Nürnberg, Experimental Renal and Cardiovascular Research, Erlangen, Germany

\section{Introduction}

Cardiovascular diseases like heart failure, arrhythmia or an ischemic stroke are the leading cause of disability for millions of people. Therefore fibrous and electrically conductive scaffolds that mimic the extracellular matrix (ECM) are of high demand for cardiac tissue engineering (CTE) [1]. The aim of this study is to develop electrically conductive Gelatin-PANi fibrous scaffolds by using the electrospinning technique for potential applications as cardiac patches.

\section{Materials \& Methods}

Aniline was either polymerized in water or directly in-situ in gelatin using both times ammonium persulfate (APS) as oxidant. A Gelatin-PANi combination was then electrospun to obtain randomly and aligned fibers, respectively, using $17.5 \mathrm{kV}, 25^{\circ} \mathrm{C}$ and $40 \%$ relative humidity as spinning parameters. The resulted fibers were investigated regarding their morphological, physicochemical and electrical properties.

\section{Results}

Uniform Gelatin-PANi fibers were produced with an alignment of over 70\%. FTIR as well as UV-VIS analyses confirmed the electrical conductive emeraldine salt form of polyaniline. 4-point-measurements showed an increased conductivity of the Gelatin-PANi combination compared to pure gelatin.

\section{Conclusion}

Aligned Gelatin-PANi fibers could be spun successfully. They showed an increased conductivity which is desired for potential CTE patches.

\section{Acknowledgement}

Financial support from DFG (Bonn, Germany) (Project BO 1191/14-1) is acknowledged.

\section{References}

[1] Chaudhuri et al., "Biomaterials and Cells for Cardiac Tissue Engineering: Current Choices", Materials Science \& Engineering C, 79 (2017) 950-957. 
S01-06

\title{
Zinc oxide as innovative nanoparticle-based drug support for the improvement of irradiation response in tumor therapy
}

\author{
*N. Wiesmann ${ }^{1}$, J. Hill ${ }^{1}$, M. Viel ${ }^{2}$, W. Tremel ${ }^{2}$, J. Brieger ${ }^{1}$ \\ ${ }^{1}$ Universitätsmedizin Mainz, HNO, Labor für Molekulare Tumorbiologie, Mainz, Germany \\ ${ }^{2}$ Johannes Gutenberg-Universität, Institut für Anorganische und Analytische Chemie, Mainz, Germany
}

Radiation therapy plays an important role in treatment of tumors but unfortunately its success is limited by the development of resistances of tumor cells to irradiation. Zinc oxide nanoparticles (ZnO-NP) were shown to exert selective cytotoxicity against tumor cells $[1,2]$ most likely via the generation of reactive oxygen species (ROS) [3]. They could become an important tool to selectively sensitize tumor cells to irradiation via nanoparticle-based targeting techniques.

Our aim was to evaluate the applicability of $\mathrm{ZnO}-\mathrm{NP}$ as radiosensitizer to improve the irradiation response of tumor cells.

We assessed cell viability after treatment with $\mathrm{ZnO}-\mathrm{NP}$ and the performance of $\mathrm{ZnO}-\mathrm{NP}$ as radiosensitizer was assessed by a colony formation assay. With the help of ZnO-NP coated by a silica-shell we evaluated their uptake into tumor cells.

We could demonstrate that $\mathrm{ZnO}-\mathrm{NP}$ exert cytotoxicity to human tumor cells, which is conveyed by dissolved $\mathrm{Zn} 2+$ ions as well as by the particles themselves. The colony formation assay showed that treatment with ZnO-NP in combination with irradiation could enhance tumor cell death and reduce clonogenic survival. $\mathrm{ZnO}-\mathrm{NP}$ could be shown to be taken up into tumor cells and premature dissolution could be prevented by embedding them into a silica coating.

All in all, the study shows that $\mathrm{ZnO}$-NPs could probably be a promising anticancer agent, to improve the irradiation response and thus clinical outcome in tumor therapy.

[1] Chandrasekaran, M. et al. (2015) In Vitro Selective Anti-Proliferative Effect of Zinc Oxide Nanoparticles Against Co-Cultured C2C12 Myoblastoma Cancer and 3T3-L1 Normal Cells. Biological trace element research.

[2] Ostrovsky, S. et al. (2009) Selective cytotoxic effect of ZnO nanoparticles on glioma cells. Nano Res., 2, 882-890.

[3] Shen, C. et al. (2013) Relating cytotoxicity, zinc ions, and reactive oxygen in $\mathrm{ZnO}$ nanoparticle-exposed human immune cells. Toxicological sciences, 136, 120-130. 


\title{
Engineering of a 3D Printable, Conductive, Alginate-based Biopolymer-Polypyrrole Composite for Electrically Active Implants
}

\author{
*T. Distler ${ }^{1}$, C. Polley ${ }^{2}$, R. Detsch ${ }^{1}$, H. Seitz ${ }^{2}$, A. R. Boccaccini ${ }^{1}$ \\ ${ }^{I}$ Institute of Biomaterials, Materials Science and Engineering, Friedrich-Alexander-University Erlangen-Nuremberg, Erlangen, Germany \\ ${ }^{2}$ Chair of Fluid Technology and Microfluidics, Faculty of Mechanical Engineering and Marine Technology, University of Rostock, Rostock, \\ Germany
}

\section{Introduction}

The development of electrically conductive materials is at key scope for muscle and nerve repair strategies. Expanding this field of application, recent studies showed beneficial effects of electrical stimulation towards cartilage and bone tissue regeneration. The treatment of complex lesion sites like osteochondral- or large-size bone defects represents a great need for customized implants of improved healing capacity. The combination of additive manufacturing techniques and the possibility of electrical stimulation through novel conductive implant materials may be a promising approach towards a new generation of sophisticated implant solutions.

\section{Objectives}

The main goal of this study was to investigate the in-situ formation of a novel, alginate-di-aldehyde (ADA) and gelatine (Gel) based-Polypyrrole hydrogel possessing electrical conductivity, manufacturability through 3D printing and biocompatibility towards the application of novel cartilage and bone repair strategies.

\section{Materials \& Methods}

Polypyrrole was in-situ polymerized inside ADA solution using oxidative polymerization by ammonium persulfate (APS). Subsequently, gelatine was added to form ADA/Gel-PPy. Different molarities of pyrrole and APS were assessed to see the effect of varying synthesis conditions on electrical conductivity, printability and processability, microstructure and stiffness. Electron beam melted (EBM) Ti6Al4V structures were coated with ADA/Gel-PPy by a dip-coating approach. The biocompatibility was assessed using ATDC-5 and MG-63 cells.

\section{Results}

It was possible to form a highly printable ADA/Gel-PPy hydrogel. The conductivity was altered by the synthesis conditions of different Py and APS molarities.

\section{Conclusion}

The hydrogel formulation introduced shows great potential towards the manufacturing of 3D printed, electrically functional hydrogel scaffolds and conductive coatings for electrically active implants.

\section{Acknowledgement}

DFG - CRC 1270 ELAINE ELectrically Active ImplaNts 


\title{
S01-08
}

\section{Alginate-laminin hydrogel blends for the cultivation of human induced pluripotent stem cell (hiPSC)-derived neural cells}

\author{
*J. Hartmann ${ }^{1}$, I. Lauria ${ }^{1}$, F. Bendt ${ }^{1}$, S. Rütten ${ }^{2}$, A. Blaeser ${ }^{3}$, E. Fritsche ${ }^{1}$ \\ ${ }^{1}$ Leibniz Research Institute of Environmental Medicine, Modern risk assessment and sphere biology, Düsseldorf, Germany \\ ${ }^{2}$ RWTH Aachen University Hospital, Institute of Pathology, Electron Microscopy Facility, Aachen, Germany \\ ${ }^{3}$ RWTH Aachen University, Biohybrid \& Medical Textiles (BioTex), AME-Helmholtz Institute for Biomedical Engineering, ITA-Institut für \\ Textiltechnik, Aachen, Germany
}

For drug and chemical compound testing human cell-based in vitro assays are needed that mimic neuronal function and better predict efficacy or toxicity. Here, functional neuronal networks that to some extent model the complexity of neuronal connectivity are warranted. Compared to rodent primary cell-based networks, production of hiPSC-derived, functionally active neuronal cultures is challenging, especially due to the long time that mixed neuronal/glia cultures take for maturing and forming functional synapses. We have succeeded in generating hiPSC-derived neuronal networks with their electrical activity detected on microelectrode arrays (MEAs). However, these two-dimensional (2D) cell culture systems exert high variability already within technical replicates and, due to their monolayer structure, only partially represent brain sophistication. To achieve higher standardization of models with improved complexity, 3D bioprinting might be a solution. Our current work studies a possible matrix for neuronal bioprinting that is cell-compatible. Relatively fast and simple tests for cell compatibility of hydrogels include evaluating hiPSC-derived neural progenitor cell migration and differentiation. Thus, we measured cell viability by mitochondrial activity, cell migration, cell differentiation by immunostainings of relevant neural markers of hiPSC-NPCs cultivated in 3D in alginate-laminin based hydrogel blends. In addition, we characterized cell-material-interactions as well as material properties such as pore size and elasticity by scanning electron microscopy and rheology, respectively. Our results suggest that alginate-laminin hydrogel blends appear as a suitable biomaterial for three-dimensional cultivation of hiPSC-derived neural cells. 


\title{
Release of biologically active proteins from modified poly ( $\varepsilon$-caprolactone) fiber mats loaded with chitosan tripolyphosphate nanoparticles allow human mesenchymal stem cells to differentiate
}

\author{
*Y. Roger ${ }^{1}$, L. Burmeister ${ }^{1}$, B. Quaas², A. L. Hoheisel ${ }^{3}$, D. de Cassan ${ }^{4}$, S. Sydow ${ }^{4}$, S. Gniesmer ${ }^{5}$, U. Rinas ${ }^{2}$, B. Glasmacher ${ }^{3}$, H. Menzel $^{4}$, A. \\ Kampmann $^{5}$, A. Hoffmann ${ }^{1}$ \\ ${ }^{I}$ Medizinische Hochschule Hannover, Orthopädie, Hannover, Germany \\ ${ }^{2}$ Leibniz Universität Hannover, Institut für Technische Chemie, Hannover, Germany \\ ${ }^{3}$ Leibniz Universität Hannover, Institut für Mehrphasenprozesse, Hannover, Germany \\ ${ }^{4}$ Technische Universität Braunschweig, Institute für Technische Chemie, Braunschweig, Germany \\ ${ }^{5}$ Medizinische Hochschule Hannover, Klinik und Poliklinik für Mund-, Kiefer- und Gesichtschirurgie , Hannover, Germany
}

Tissue transitions like tendon bone junctions (enthesis) consist of a variety of features including a gradient of properties with specific characteristics e.g. gradients in structure as well as composition and mechanical characteristics. At present there are limitations of implants with respect to such important adjustments.

Electrospun poly ( $\varepsilon$-caprolactone) (PCL) fiber mats are a promising material for this kind of tissue engineering as they can be modified using a chitosan grafted with PCL which allows further modifications with nanoparticular drug-delivery systems and improves cyto- and biocompatibility. Furthermore the electrospinning process can be changed from aligned to random spinning of the fibers which allows a gradient of fiber directions throughout the fiber mats thus mimicking the tissue transition. In order to achieve a controlled delivery of bioactive factors such as drugs or proteins to a specific region, nanoparticles are a promising option. Here we used Chitosan/Tripolyphosphate nanoparticles loaded with recombinant (E. coli) human TGF- $\beta 3$ and BMP-2. The transforming growth factor superfamily, including TGF- $\beta$ s and BMPs, is involved in various cellular processes such as proliferation, differentiation or development. The release of TGF- $\beta 3$ from the nanoparticle-coated fiber mats induced chondrogenesis in human bone marrow-derived mesenchymal stem cells (MSCs). Similarly the release of BMP-2 from nanoparticle-coated fiber mats induced osteogenic features in human bone marrow-derived mesenchymal stem cells. These findings make modified fiber mats in combination with the release of relevant factors a promising candidate for implants bridging complex tissue transitions. 


\section{D plotting of anatomically relevant structures from calcium phosphate cements with methylcellulose as support material}

*T. Ahlfeld ${ }^{1}$, D. Kilian ${ }^{1}$, T. Köhler ${ }^{1}$, C. Czichy ${ }^{2}$, L. Mika ${ }^{3}$, P. Sembdner ${ }^{3}$, P. Korn ${ }^{4}$, S. Odenbach ${ }^{2}$, R. Stelzer ${ }^{3}$, A. Lode ${ }^{1}$, M. Gelinsky $^{1}$ ${ }^{I}$ Technische Universität Dresden, Centre for Translational Bone, Joint and Soft Tissue Research, Dresden, Germany

${ }^{2}$ Technische Universität Dresden, Institute of Fluid Mechanics, Chair of Magnetofluiddynamics, Measuring and Automation Technology,

Dresden, Germany

${ }^{3}$ Technische Universität Dresden, Institute of Machine Elements and Machine Design, Chair of Machine Design and CAD, Dresden, Germany

${ }^{4}$ Charité Universitätsmedizin Berlin, Klinik für Mund-, Kiefer und Gesichtschirurgie, Berlin, Germany

In the past, many groups investigated the application of different additive manufacturing technologies to fabricate hydroxyapatite (HAp) implants for bone repair. In recent studies, our group showed that low temperature extrusion, 3D plotting, of calcium phosphate cements (CPC) allowed integration of growth factors and cells into the fabrication process, which opens new options to enhance bone regeneration and resorption kinetics of the implant [Lode et al., 2014]. However, 3D plotting is limited in terms of shape diversity, as overhangs, cavities or a three-dimensional profile (instead of a flat basic area) cannot be realized readily.

Herein we demonstrate that anatomically relevant structures can be plotted from CPC by usage of methylcellulose (mc) as support material. First, 6,8 and $10 \mathrm{wt} \% \mathrm{mc}$ were systematically evaluated for rheological and extrusion properties. All three hydrogels displayed shear-thinning behaviour and fast shear recovery. As filament fusion tests showed highest shape fidelity for $10 \mathrm{wt} \% \mathrm{mc}$, it was chosen as suitable support ink.

Multi-channel plotting of CPC and $10 \mathrm{wt} \%$ mc enables precise fabrication of critical overhangs and cavities, that was proven with a flipped vs. a standard pyramid and a cube-inside-cube-structure as model structures. After setting of CPC, constructs were incubated in water overnight at 4 ${ }^{\circ} \mathrm{C}$ to dissolve mc, leaving behind the HAp structures without mc residuals. Micro computed tomography evidenced the cavity within the HAp cube.

Fabrication of anatomical relevant shapes was investigated using the hand scaphoid bone as example. CT data from a patient were converted to a 3D model of a hand and the scaphoid was extracted to a 3D stl-file and plotted with CPC and mc as support material. After CPC setting and mc dissolution, the resulting construct comprised high shape and dimensional fidelity. Finally, we have fabricated a patient-individual HAp implant perfectly fitting into a critical defect of a maxillary (fig 1).

\section{Figure 1}
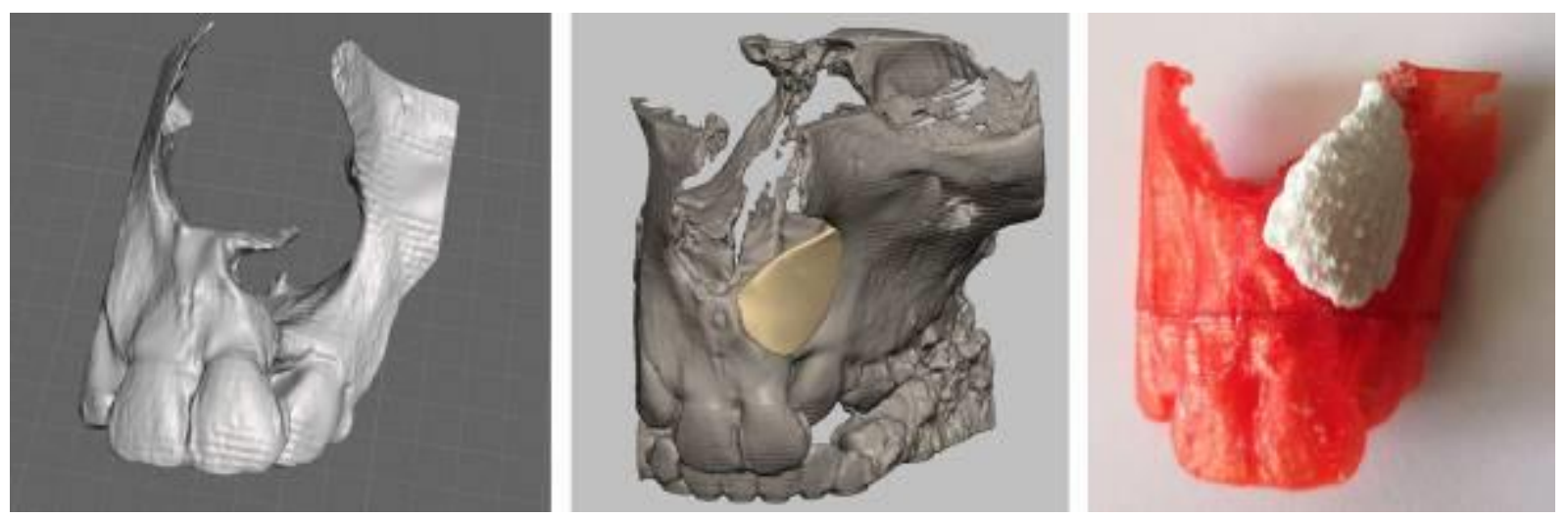


\title{
Efficacy of electrochemical attack to treat biofilms on (dental) titanium implants
}

\author{
*F. Kaiser ${ }^{1}$, D. Scharnweber ${ }^{1}$, C. Wolf-Brandstetter ${ }^{1}$ \\ ${ }^{I}$ Institute of Materials Science, Max Bergmann Center of Biomaterials, TU Dresden, Dresden, Germany
}

\section{Introduction}

Titanium based dental implants are used for decades with high success rates. However, since such implants can get in partial contact with bacteria of the oral cavity there is a permanent risk of contamination leading to inflammatory reactions resulting in degradation of bone and loss of implants in the worst case - the so called peri-implantitis (PI). So far no non-surgical therapy is able to eradicate PI. Aim of this study is to develop a minimal invasive therapy to maintain the implant. The most important goal is the efficient killing of adherent bacteria that are not accessible by other non-invasive techniques.

\section{Experimental Methods}

A strain of genetically modified E.coli with green fluorescent protein (GFP) and specific for biofilm formation in minimal medium was used [1]. Bacteria were grown up to the exponential phase and seeded on top of commercially pure titanium disks for 60 min and then cultured under dynamic cultivation in biofilm medium for further $17 \mathrm{~h}$. Electrochemical treatment in phosphate buffered saline included variable anodic, cathodic, or anodic and cathodic polarization regimes. Electrochemical impedance spectroscopy was used to characterize bacterial colonization before and after electrochemical treatment. Additionally, bacteria were stained with LIVE/DEAD ${ }^{\circledR}$ BacLight ${ }^{\mathrm{TM}}$ Bacterial Viability Kit and examined with fluorescence microscopy.

\section{Results and discussion}

The fluorescence images and impedance data show increasing efficiency in bacteria killing with increasing current density. Current densities of $0.5 \mathrm{~mA} / \mathrm{cm}^{2}$ and below (Fig. 1, A and B) kill bacteria only partly, whereas a treatment with $1 \mathrm{~mA} / \mathrm{cm}^{2}$ and above (Fig. 1, C and D) kills almost all bacteria. The results indicate that reactive chlorine and oxygen species generated during the electrochemical treatment, the amount of which depends on the current density, are the main reason for the observed antibacterial effect.

\section{References}

[1] Reisner et al. Mol Microbiol 48(4): 933-946, 2003.

\section{Figure 1}
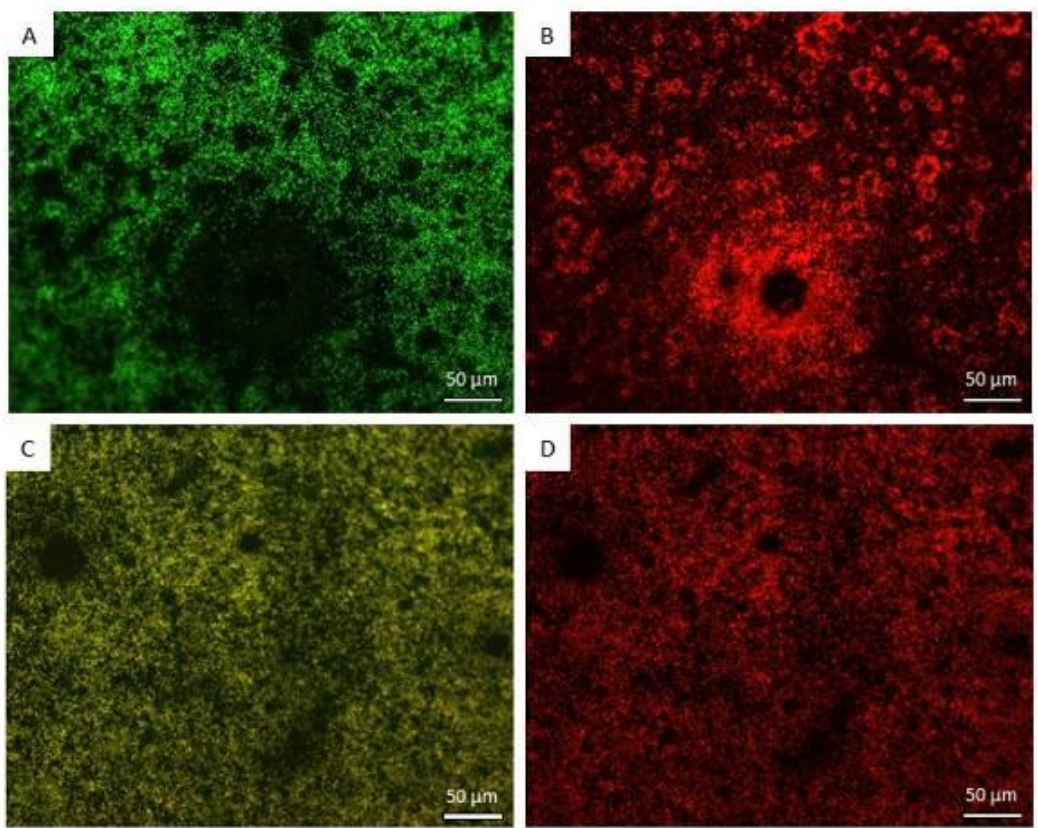

Fig 1 Fluorescence images of samples treated with $0.25 \mathrm{~mA} / \mathrm{cm}^{2}$ (A: green fluorescence, $\mathrm{B}$ : red fluorescence) and $1 \mathrm{~mA} / \mathrm{cm}^{2}$ (C: green fluorescence, D: red fluorescence) with cathodic and anodic polarization 


\section{S01-13}

\section{Low-temperature-sterilization method for biomaterials}

*D. Voigt ${ }^{1}$, M. Meyer ${ }^{1}$

${ }^{I}$ Forschungsinstitut für Leder und Kunststoffbahnen gGmbH, Biomaterials/Collagen, Freiberg, Germany

\section{Introduction}

There is an increasing interest on new sterilization techniques for biological materials, due to the extension of collagen scaffold usage for reconstructing various tissues. Preservation of the collagen scaffold structures is important with respect to its biocompatibility and stability. Standard sterilization methods using heat or steam are unfavourable, because they cause instability of the collagen scaffold as a result of thermal denaturation. Established sterilization methods like $\gamma$-rays [1] need an ambitious technical equipment and have a destructive effect on the molecular structure of collagen. Methods utilizing ethylene oxide as reagent are disadvantageous too, due to its toxicity and necessity to detoxify the sterilized material [2].

\section{Methods}

We present a new sterilization method for collagen utilizing $\mathrm{H}_{2} \mathrm{O}_{2}$ as reactive reagent. The properties of the collagen foil and their structural modifications were investigated with regard to the variation of $\mathrm{H}_{2} \mathrm{O}_{2}$ concentrations, the duration of exposure to $\mathrm{H}_{2} \mathrm{O}_{2}$ and plasma treatment.

\section{Results}

The figure shows that $\mathrm{H}_{2} \mathrm{O}_{2}$-plasma does not lead to marked degradation, while g-rays markedly affect the collagen structure.

Figure: Size exclusion chromatograms of collagen foil (a) before sterilization, (b) after $\mathrm{H}_{2} \mathrm{O}_{2}$-plasma sterilization and (c) after gamma-ray sterilization. The numbers reflect crosslinked material (p), the dimers of collagen chains $(\beta)$ and single strands $(\alpha)$. Gamma treatment leads to randomly degraded collagen peptides [3].

\section{Conclusion}

The low-temperature sterilization with $\mathrm{H}_{2} \mathrm{O}_{2}$-plasma is a promising alternative to conventional sterilization methods.

We acknowledge the funding of this project by the BMWi (INNO-KOM Ost).

[1] E. M. Noah et al., Biomaterials, vol. 23, no. 14, pp. 2855-2861, Jul. 2002.

[2] M. J. Doherty et al., Biomaterials, vol. 14, no. 13, pp. 994-998, Oct. 1993.

[3] M. Meyer et al., Biomacromolecules, vol. 4, no. 6, pp. 1727-1732, Nov. 2003.

\section{Figure 1}

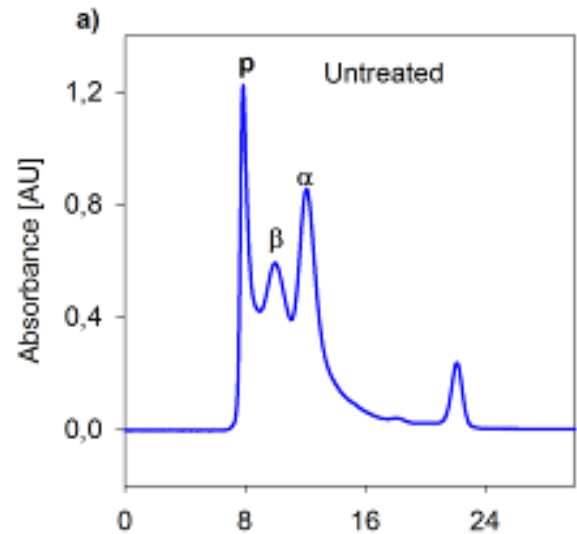

b)

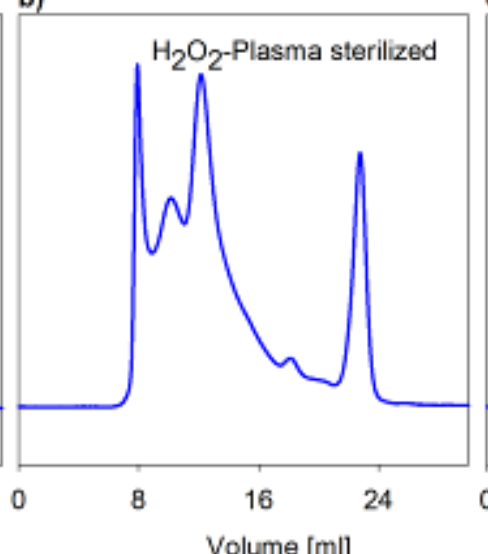

c)

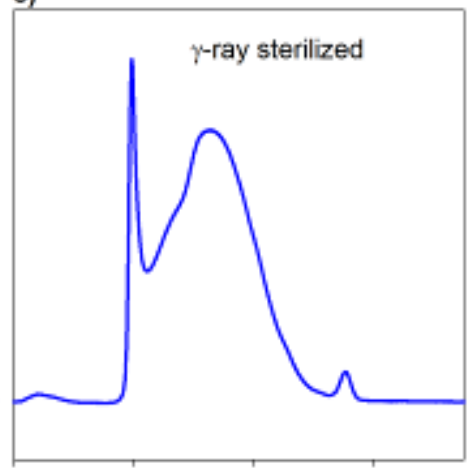




\title{
S01-15
}

\section{Bioactive and antibacterial PCL/Rifampicin/HA nanofibrous surface coatings on Titanium for implant-associated infections}

\author{
*S. K. K. Anugari ${ }^{1,2,3}$, A. Kizhakeyil ${ }^{4}$, R. Rahul ${ }^{2}$, M. Doble ${ }^{3}$, T. S. Kumar ${ }^{1}$, R. Seeram ${ }^{2}$ \\ ${ }^{I}$ Indian Institute of Technology Madras, Medical Materials Laboratory, Department of Metallurgical and Materials Engineering, Chennai, India \\ ${ }^{2}$ National University of Singapore, Department of Mechanical Engineering, Singapore, Singapore \\ ${ }^{3}$ Indian Institute of Technology Madras, Bhupat and Jyoti Mehta School of Biosciences, Department of Biotechnology, Chennai, India \\ ${ }^{4}$ Nanyang Technological University, Lee Kong Chian School of Medicine, Singapore, Singapore
}

\section{Introduction}

Titanium (Ti) and Ti alloys are the material of choice for orthopedic implants because of their good biological and reliable mechanical properties. However, these biomedical alloys are prone to implant-associated infections due to microbial adhesion/biofilms, subsequently leading to implant failure. To avoid such failures, precautionary measures are essential to mitigate implant-related infections in early stages of implantation. One of the main strategies to prevent such infections is by using antibacterial drugs on the implant surfaces.

\section{Objectives}

The objective behind current research is to develop an organic-drug-inorganic hybrid scaffolds with known antibacterial properties of rifampicin (RIF), bioactivity properties of hydroxyapatite (HA) and mechanical properties of the poly ( $\varepsilon$-caprolactone) (PCL) to modify the surface of Ti implants.

\section{Materials \& Methods}

RIF, an effective antibacterial drug proved its potential against the bacteria causing bone infections. HA, an osteoconductive ceramic, have been verified to be a significant material for bioactivity enhancement. In recent years, electrospinning has been widely recognized as one of the most efficient technologies to fabricate micro/nanosized fibres for bone tissue engineering applications, especially with a polymer, PCL.

\section{Results}

PCL/RIF/HA nanocomposites with different concentrations were fabricated by electrospinning technique. Characterization techniques such as SEM, EDS, XRD, FITR were used to analyse PCL/RIF/HA nanocomposites. Cellular interactive responses such as adhesive and proliferation were evaluated using hFOB human fetal osteoblast cell lines. The inhibition efficiency for the nanocomposites against s.aureus bacteria was determined by the bacterial viability test.

\section{Conclusions}

PCL/RIF/HA nanocomposite fibres were fabricated by means of $8 \%$ PCL, $10 \%$ HA and RIF for bactericidal efficacies. The fabricate scaffolds are effective in inhibiting bacterial growth on $\mathrm{Ti}$ implants.

Figure 1

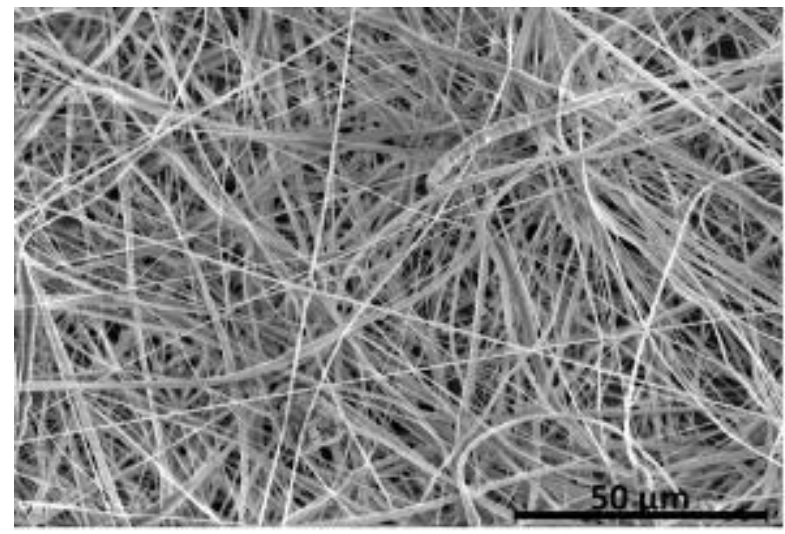

PCL Nanofibers

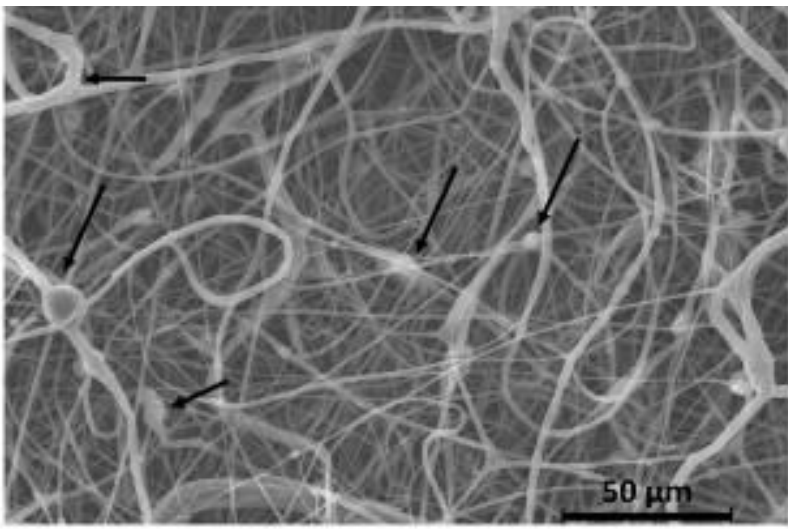

PCL/RIF/HA Nanocomposite Fibres 


\title{
S01-16
}

\section{In vitro evaluation of biofilm-inhibiting compounds to treat infective endocarditis}

\author{
*V. Pauker ${ }^{1}$, D. Gottschling ${ }^{1}$, L. Maletzki ${ }^{2}$, A. Strohbach ${ }^{2}$, S. Kohse ${ }^{3}$, S. Petersen ${ }^{4}$, N. Grabow ${ }^{3}$, K. Riedel ${ }^{1}$ \\ ${ }^{\prime}$ Institute of Microbiology, University of Greifswald, Greifswald, Germany \\ ${ }^{2}$ Forschungscluster III, University Hospital Greifswald, Greifswald, Germany \\ ${ }^{3}$ Institute for Biomedical Engineering, University Hospital Rostock, Rostock, Germany \\ ${ }^{4}$ Faculty of Engineering and Computer Science, University of Apllied Science, Osnabrück, Germany
}

Infective endocarditis, short IE, still poses a serious risk after surgical valve replacement. Especially, after transcatheter aortic valve implantation (TAVI), which is commonly used as treatment of aortic stenosis, the rates of early IE can be as high as $3.03 \%$. The major cause of IE are bacteria of the genera Streptococcus, Staphylococcus and Enterococcus, which colonize the implants and are able to form biofilms. Biofilms are characterized by cells embedded in a slimy matrix composed of extracellular polymeric substances (EPS) such as polysaccharides, proteins, eDNA, and amyloid fibers. The EPS acts as a physical barrier between bacterial cells and environmental stress, including osmotic stress, heat, starvation, antibiotics and antibodies. Infections of cardiovascular implants can lead to life threatening conditions and prevention of infection and especially biofilm formation is a necessary prerequisite for an effective treatment. In order to determine the capability of different compounds to interfere with biofilm growth, a standard micro-titer assay and a flow-through system will be employed for a quantitative and qualitative evaluation of the biofilm forming capacity of the above-mentioned Gram-positive cocci. As a starting point, $\mathrm{N}$-acetylcysteine and chlorhexidine were tested to prevent formation and dissolve existing Staphylococcus aureus biofilms. The compounds will be further evaluated as possible coating substances for endothelialized TAVI surfaces. 


\title{
S01-17
}

\section{Non-linear imaging as tool in biomaterial research}

\author{
*D. Thieme ${ }^{1}$, T. Fuchsluger ${ }^{2}$, D. Schubert ${ }^{1}$ \\ ${ }^{1}$ University Erlangen-Nuremberg, Institute of Polymer Materials, Erlangen, Germany \\ ${ }^{2}$ University Erlangen-Nuremberg, Ophthalmology, Erlangen, Germany
}

\section{Introduction}

In the past material research focused mostly on the toxicity of the used polymers, the solvent or the processes used to produce biomaterials. In the recent years biological factors like surface modifications, biocompatibility or degradation complemented this profile in biomaterial production. Step by step biomaterial engineering reached the goal of a transplantable scaffold - raising the question, how the fate of a transplanted biomaterial can be followed in situ.

The future development of a transplanted construct in vivo is the crucial point in tissue engineering, especially with focus on characteristics of used cell populations and biodegradation.

Non-linear imaging is an emerging field in biological research combining highly sensitive detectors with tissue penetrating low wavelength laser systems. This system is capable of imaging cells and structures at confocal resolution within the tissue until a depth of $2 \mathrm{~mm}$. With this technology it seems possible to follow the future developmen of a biomaterial in situ for the first time.

\section{Objectives}

Non-linear imaging is introduced as a versatile tool for the imaging of biomaterials and human tissues. Second harmonic generation will be evaluated as core feature to investigate characteristics of tissue and biomaterial alike.

\section{Material and Methods}

Human donor corneas and Polycaprolactone-based biomaterials are used as materials. Non-linear imaging was done with a Zeiss LSM880 NLO fitted with non-descanned detectors coupled to a two-photon Spectra Physics DeepSee Laser for two-photon excited fluorescence.

\section{Results}

We can demonstrate that non-linear imaging is feasible for whole tissue imaging of human corneas and for structural analysis of the tissue and the biomaterials.

\section{Conclusions}

With non-linear imaging we can evaluate the fate of a transplanted biomaterial in situ. In addition, second harmonic generation is a unique feature of the method enabling new insights in tissue und biomaterial properties. 


\title{
S01-18
}

\section{Plant virus nanoparticles embedded in hydrogel matrices induce biomineralization and human mesenchymal stem cells osteogenic differentiation}

\author{
*Y. Y. Lin ${ }^{1}$, J. Röder ${ }^{2}$, C. Dickmeis ${ }^{2}$, S. Rütten ${ }^{3}$, U. Commandeur ${ }^{2}$, H. Fischer ${ }^{1}$ \\ ${ }^{I}$ RWTH Aachen University Hospital, Dental Materials and Biomaterials Research, Aachen, Germany \\ ${ }^{2} R W T H$ Aachen University, Institute for Molecular Biotechnology, Aachen, Germany \\ ${ }^{3}$ RWTH Aachen University Hospital, Electron Microscopy Facility, Aachen, Germany
}

To develop bone replacements, hydrogels are widely used to mimic the extracellular matrix. However, they often lack biochemical cues to modulate the cellular response. Hence, peptide fusion can be applied to improve the bioreactivity. Potato virus X (PVX), a flexible rod-shaped plant virus, can be genetically engineered to present more than a thousand peptides on its surface.

PVX was modified to present polyglutamate and hydroxyapatite-binding peptides, both of which are reported as potent nucleators for mineralization. To enhance cell adhesion, PVX was also engineered to present the integrin-binding motif (RGD). We hypothesized that biomineralization and osteogenic differentiation of human mesenchymal stem cells (hMSCs) can be stimulated by incorporating modified PVX on $2 \mathrm{D}$ surfaces and in 3D hydrogels.

The interaction between PVX and hMSCs were first examined on 2D surfaces by culturing hMSCs on PVX-coated coverslips, and then both were embedded into 3D hydrogels. PVX coating was characterized by immunostaining. Live/dead assay was performed in both 2D and 3D cultures. hMSCs osteogenic differentiation was induced on PVX-coated surfaces. In addition, scanning electron microscopy was used to examine the mineralization effect after PVX-hydrogels were immersed in simulated body fluid.

Fluorescence measurement showed a high coating efficiency of PVX on 2D surfaces. PVX-E8 and PVX-RGD had a positive effect for cell attachment on 2D surfaces. High cell viabilities in 2D and 3D culture exhibited excellent cytocompatibility of the PVX nanoparticles. Moreover, hMSCs osteogenesis was verified on 2D tailored surfaces by Alizarin red staining. Furthermore, PVX-HABP and PVX-RGD showed significant impact on hydroxyapatite nucleation in the 3D hydrogels. In conclusion, the genetically modified PVX nanoparticles showed excellent hMSC attachment, viability, and osteogenesis in $2 \mathrm{D}$ and $3 \mathrm{D}$ cultures, and offer great potential as a new hydrogel composition for bone tissue engineering. 


\section{Development of a hydrogel-based vocal fold tissue phantom for OCT imaging and laser ablation}

*C. Bärhold ${ }^{1}$, J. Napier ${ }^{2}$, M. Zabic ${ }^{3}$, M. Ptok ${ }^{1}$

${ }^{I}$ Medical School Hannover, Department of Phoniatry and Paediatric Audiology, Hannover, Germany

${ }^{2}$ University of Applied Sciences Emden, Institute for Laser and Optics, Emden, Germany

${ }^{3}$ Leibniz Universität Hannover, Institute of Quantum Optics, Hannover, Germany

Artificial phantoms that reproduce optical and mechanical properties of living tissue are useful for the development of new surgical instruments and receive wide spread attention in the research field of biomedicine [1]. Of particular interest in this field is the development of new biomaterials, e.g. nanocomposite materials [2] or the optimization of currently available materials with additives that have a specific response similar to the tissue parameters.

Our current research focusses on the development and fabrication of an artificial vocal fold tissue phantom to provide a realistic model for laser surgery and contactless imaging with optical coherence tomography (OCT). The hydrogel phantom is prepared by using poly(ethylene glycol) diacrylate (PEGDA) and PEG dimethacrylate (PEGDMA) with different mixing ratios $(2.5,5,10,25 \mathrm{wt} \%)$ to produce a soft, non-brittle hydrogel. Clay powder (Laponite XLG) is added to improve the mechanical properties of the hydrogel. The cross-linking is conducted by in situ radical polymerization with TEMED and APS as catalysts. The multi-layered structure is created with a sequential casting process using predefined molds that match the human vocal fold dimensions.

For the laser ablation of the artificial vocal folds a thulium laser (center wavelength: 1940nm) is used. The cutting depth and width is compared with cuts on porcine vocal folds, which have similar laser tissue interaction characteristics to human vocal folds.

Further research is aimed to improve the elasticity of the materials as well as optimising the physical and optical properties, e.g. the refractive index, for both OCT imaging and laser ablation by adding natural and synthetic additives.

Figure 1: Laser ablation on an artificial vocal fold hydrogel, coronal view

[1] Lamouche et al. Biomedical optics express 3.6 (2012): 1381-1398.

[2] Chang et al. Soft Matter 6.20 (2010): 5157-5164.

Figure 1

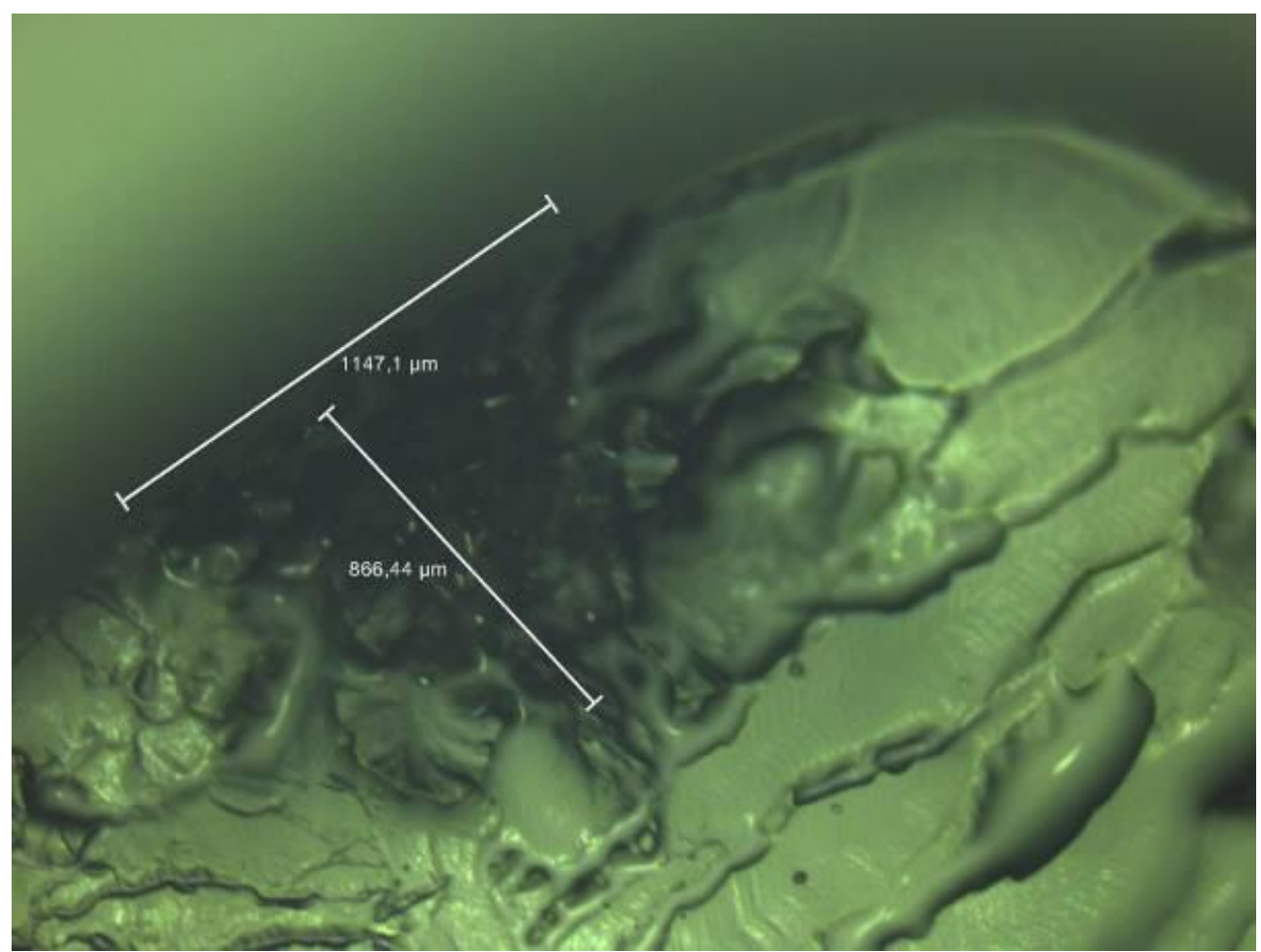


S01-20

\title{
Simulation of the tumor ECM using 2PP generated Scaffolds based on (D, L)-Lactide- $\varepsilon$-Caprolactone Copolymers
}

\author{
*N. Hauptmann ${ }^{1}$, J. Ludolph ${ }^{1}$, H. Rothe ${ }^{1}$, K. Liefeith ${ }^{1}$ \\ ${ }^{1}$ Institute for Bioprocessing and Analytical Measurement Techniques e. V., Biomaterials, Heilbad Heiligenstadt, Germany
}

\section{Introduction}

Tumor cells respond to changes in the stiffness of their environment by cytoskeletal remodeling. A stiffening of the ECM leads to a more invasive phenotype which results in metastasis. Therefore, three-dimensional cell-culturing systems were established to analyze cell-matrix interactions. For the fabrication of these scaffolds, rapid prototyping technologies are usually used, under which the 2-Photonpolymerisation (2PP) is the most promising one because of the excellent resolution below $100 \mathrm{~nm}$.

\section{Objectives}

Within this study, we established a cell-culturing platform with tumor specific stiffness properties for analyzing the effect of matrix stiffness on the proliferation and morphology of tumor cells.

\section{Materials \& Methods}

Scaffolds based on (D, L)-Lactide- $\varepsilon$-Caprolactone copolymers with different stiffness were fabricated by $2 \mathrm{PP}$. Then the influence of the matrix elasticity on the remodeling of the cytoskeleton was measured by colloidal force spectroscopy. 2PP generated scaffolds were seeded with tumor cells and cellular proliferation and morphology were analyzed.

\section{Results}

We successfully established a 3D cell-culturing system by $2 \mathrm{PP}$ with tumor specific mechanical properties. Tumor cells responded to the matrix elasticity by cytoskeletal reorganization. Proliferation studies with invasive and non-invasive tumor cells showed an increase in the number of cells over the whole cultivation time. Furthermore, cells proliferate in the third direction and start to remodel the scaffold material.

\section{Conclusion}

In summary, we established a highly biocompatible artificial Tumor-ECM with defined stiffness properties by $2 \mathrm{PP}$. We could show that cells colonize the whole scaffold and proliferate inside the scaffold material. The results showed the great potential of the copolymer for simulation of the tumor specific ECM.

Figure: A) CAD model of scaffold structure; B) CLSM image of a 2PP Scaffold; C) MCF-7 cells inside 2PP scaffold (blue: nucleus, green: CD44, red: actin)

\section{Figure 1}

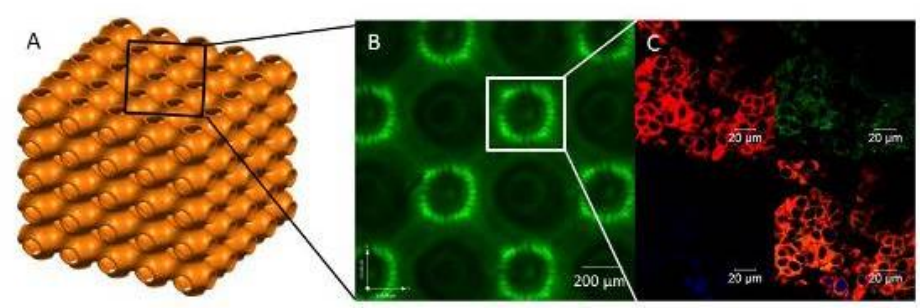

